Re-submitted to: $\quad$ Bioresource Technology (BITE-D-10-04735)

Date:

15 January 2011

\title{
Change in the Fouling Propensity of Sludge in Membrane Bioreactors (MBR) in Relation to the Accumulation of Biopolymer Clusters
}

\author{
Fei-yun Sun ${ }^{\mathrm{a}, \mathrm{b}}$, Xiao-mao Wang ${ }^{\mathrm{a}}$, Xiao-yan $\mathrm{Li}^{\mathrm{a} *}$ \\ ${ }^{a}$ Environmental Engineering Research Centre, Department of Civil Engineering \\ The University of Hong Kong, Pokfulam Road, Hong Kong, China \\ ${ }^{\mathrm{b}}$ Harbin Institute of Technology Shenzhen Graduate School, Shenzhen 518055, China \\ (*Corresponding author: phone: 852-28592659; fax: 852-28595337; e-mail: xlia@hkucc.hku.hk)
}

\begin{abstract}
A membrane bioreactor (MBR) and an activated sludge process (ASP) were operated side by side to evaluate the change of sludge supernatant characteristics and the evolution of the sludge fouling propensity. The MBR sludge had a higher organic concentration and more biopolymer clusters (BPC) in the supernatant compared with ASP. BPC increased in both concentration and size in the MBR. The results show that the change in the liquid-phase property had a profound effect on the sludge fouling propensity. MBR operation transformed typical activated sludge to MBR sludge with a higher fouling propensity. Distinct from the ASP, membrane filtration retained soluble microbial products (SMP) within the MBR, and the vast membrane surface provided a unique environment for the transformation of SMP to large size BPC, leading to further sludge deposition on the membrane surface. Thus, membrane filtration is the crucial cause of the inevitable fouling problem in submerged MBRs.
\end{abstract}


Keywords: Activated sludge process; Biopolymer clusters (BPC); Membrane fouling; Membrane bioreactor (MBR); Biological wastewater treatment.

\section{Introduction}

Membrane bioreactors (MBRs) have become an increasingly attractive option in wastewater treatment and water reclamation where an excellent and stable effluent quality is required (Judd, 2006; Drews, 2010). Distinct from the conventional activated sludge process (ASP), an MBR ensures complete sludge-effluent separation and produces effluent that contains much fewer colloidal substances through the use of membrane filtration, which is a better barrier to fine particles (Drews, 2010). Moreover, MBRs allow a high biomass concentration and the complete uncoupling of sludge age and hydraulic retention time. As a result, they have a smaller footprint, produce less excess sludge, and allow the more efficient removal of organic pollutants and nutrients than the ASP. However, the reduction of membrane permeability caused by membrane fouling, a phenomenon described as the accumulation of foulants on or in the membrane, is still the major obstacle to the widespread application of MBRs in biological wastewater treatment (Judd and Jefferson, 2003).

Common approaches to the alleviation of membrane fouling in MBRs include operations to keep the filtration flux below a critical value, increasing the aeration intensity for membrane surface cleaning, and the adoption of frequent membrane backwashing (Meng et al., 2009; Judd and Jefferson, 2003; Yang et al., 2010). Nevertheless, membrane fouling, primarily in the form of sludge layer deposition on the membrane surface, still remains largely inevitable (Drews, 2010). Efforts have been made to understand the high fouling propensity of MBR sludge from several aspects (Le-Clech et al., 2006; Meng et al., 2009). It was originally believed that sludge concentration (viscosity) was the key factor that determined the membrane-fouling rate (Magara and Itoh, 1991). Later, the abundance of 
extracellular polymeric substances (EPS) in the sludge was considered to be the main cause of membrane fouling (Nagaoka et al., 1996; Wang et al., 2010; Wu and Lee, 2011). In recent years, the contribution of the liquid-phase property of the sludge, such as the colloidal and organic matter in the supernatant, to membrane fouling development was recognized (Defrance et al., 2000; Judd, 2006), and apparent correlations have been reported between the liquid-phase organic concentration of sludge and the membrane-fouling rate (Drews et al., 2008; Drews, 2010). Comparisons of the MBR and ASP (Shin and Kang, 2003; Masse et al., 2006), provide further evidence of organic accumulation in the MBR suspension, and demonstrate that MBR sludge has a consistently higher organic content in the supernatant than in the permeate (Chu and $\mathrm{Li}, 2005$; Wang and Li, 2008). Nonetheless, the crucial difference between MBR sludge and conventional activated sludge has yet to be identified with respect to their respective membrane-fouling propensities.

In activated sludge, soluble microbial products (SMP) are the major organic components in the supernatant (Laspidou and Rittmann, 2002). However, SMP, in their nascent soluble form, are unlikely to be the primary foulants, as they mostly pass through the microfiltration or ultrafiltration membranes used in an MBR. Recent research indicates that MBR sludge contains a pool of free organic solutes termed biopolymer clusters (BPC) that can be much bigger than SMP (Wang et al., 2007; Sun et al., 2008; Lin et al., 2009). BPC differ from biomass in that the former are mainly composed of abiotic polymers, rather than microorganisms. The effect of liquid-phase organics on membrane fouling is largely due to the detrimental role of BPC in fouling layer formation (Wang and $\mathrm{Li}$, 2008). It appears that the large membrane surface of an MBR provides a suitable environment for BPC formation and growth, which is not the case for the ASP. However, most previous studies have overlooked the presence of BPC and its impact on the fouling property of MBR sludge. 
Moreover, it is still unclear whether and how activated sludge is transformed into a BPC-rich MBR sludge by the MBR process.

In this study, comparative experiments were conducted on the membrane-fouling propensity of typical activated sludge and MBR sludge. A submerged MBR and an ASP were operated side by side under the same conditions. Two types of seed biomass from an MBR and an ASP system were used as the seed sludge, dividing the experimental study into two separate but closely related stages. The evolution of the fouling propensity of the sludge from the two reactors during the startup was evaluated with a focus on changes in the liquidphase property. The aims of the study were (1) to investigate the effect of organic matter, particularly BPC, in the supernatant on the fouling propensity of sludge, (2) to demonstrate the retention of SMP and the accumulation and growth of BPC in an MBR system, and (3) to reveal the transformation from a typical activated sludge to MBR sludge by MBR operation and the resulting change in the fouling property. The research findings should improve the fundamental understanding of the membrane-fouling problem in MBRs.

\section{Materials and Methods}

\subsection{Experimental setup and operation}

An ASP system and a submerged membrane bioreactor (MBR) were operated side by side for comparison. The ASP setup consisted of an aeration tank (10 L in working volume, $14 \mathrm{~cm}$ in diameter, and $120 \mathrm{~cm}$ in height) functioning as a bioreactor and a settling column (4.6 L in working volume, $9 \mathrm{~cm}$ in diameter, and $100 \mathrm{~cm}$ in height). Aeration was conducted through an air diffuser at the bottom of the aeration tank, and the aeration rate was kept more than $3.7 \mathrm{~L} / \mathrm{min}$, resulting an aeration intensity (superficial air flow velocity) of about $1 \mathrm{~cm} / \mathrm{s}$. The settled sludge in the settling column was returned to the aeration tank by a peristaltic 
pump (MasterFLEX, Cole-Parmer) at a recirculation rate of around 50\% of the influent flow, and the overflow of the supernatant was discharged as the effluent. The hydraulic retention time (HRT) and sludge retention time (SRT) of the ASP reactor were maintained at $6 \mathrm{~h}$ and 9 d, respectively (Table 1).

The MBR resembled the ASP except that the sludge sedimentation was replaced by membrane filtration. The MBR consisted of an aeration tank that had a working volume of 5 $\mathrm{L}$ and an immersed 0.4- $\mu \mathrm{m}$ polyethylene hollow-fiber membrane module (surface area $=0.2$ $\mathrm{m}^{2}$, Mitsubishi Rayon). The effluent was drawn through the membrane by a suction pump during filtration at a rate set to keep the HRT identical to that of the ASP reactor, and the flux adopted was $0.1 \mathrm{~m} / \mathrm{d}$. A filtration to relaxation time ratio of $18: 2 \mathrm{~min}$ was applied. As the aeration rate have significant effect onto the characteristics of sludge flocs (Ji and Zhou, 2006), the same aeration intensity as the ASP system at about $1 \mathrm{~cm} / \mathrm{s}$ was kept, which was provided at the bottom of the reactor for the shear cleaning of the membrane surface. The trans-membrane pressure (TMP) was monitored by a manometer in kPa transformed from mm Hg. Once the membrane had become seriously fouled (when the TMP reached around 80 $\mathrm{kPa}$ ), it was washed thoroughly with running tap water to restore its permeability for a new cycle of MBR operation (Chu and Li, 2005; Sun et al., 2008).

Both the ASP and the MBR were fed with the same glucose-based synthetic wastewater, which was prepared according to the basic recipe given in the Environmental Engineering Process Laboratory Manual of the AEESP (2001). In addition, actual domestic sewage collected from the Stanley Sewage Treatment Works in Hong Kong was dosed into the substrate feed to supply around $10 \%$ of the total organic load. The influent had a chemical oxygen demand (COD) of around $500 \mathrm{mg} / \mathrm{L}$ and a COD:N:P ratio of about 100:9:3. $\mathrm{NaHCO}_{3}$ was added to the influent at $50 \mathrm{mg} / \mathrm{L}$ or greater to maintain the $\mathrm{pH}$ of the solution in the bioreactors at between 6.5 and 7.5. The other experimental conditions, including the food-to- 
microorganisms $(\mathrm{F} / \mathrm{M})$ ratio, for the two systems are summarized in Table 1 . Under these process conditions, the sludge concentrations measured as mixed liquor suspended solids (MLSS) were kept at a level of around $5 \mathrm{~g} / \mathrm{L}$ in both reactors. The reactors were operated at room temperature $\left(22-25^{\circ} \mathrm{C}\right)$, and the water temperature was $20-22^{\circ} \mathrm{C}$.

\subsection{Experimental design and sludge and liquid samples}

Two experimental stages, Experiment I and Experiment II, were defined with respect to the seed biomass with which the bioreactors were inoculated (Table 1). In Experiment I, both the MBR and ASP reactors were seeded with sludge obtained from an MBR system that had already been operating for more than four years (Wang et al., 2007, Sun et al., 2010). In Experiment II, activated sludge collected from a regional wastewater treatment plant (Stanley Wastewater Treatment Works, Hong Kong) was used as the seed sludge. Each experimental stage lasted for around $70 \mathrm{~d}$, or more than seven SRTs, to ensure the complete startup of the bioreactors. During the experiments, the effluent and suspension in each reactor were sampled three times a week. The supernatant of the sludge suspension was then obtained through the sedimentation of the sludge $(50 \mathrm{~mL})$ for $4 \mathrm{~h}$ at $4^{\circ} \mathrm{C}$. The liquid samples, including the effluent and sludge supernatant, were analyzed for turbidity and viscosity, and for total organic carbon (TOC) content and its protein (PN), polysaccharide (PS), and humic substance (HS) components.

Additionally, sludge was sampled regularly from the bioreactors and its Bound-EPS content analyzed. The sludge sample was first centrifuged at 4,000 $\mathrm{g}$ for $5 \mathrm{~min}$ and the supernatant decanted. The sludge pellet was then re-suspended with a $0.05 \% \mathrm{NaCl}$ solution to the original volume (50 mL) with the aid of a vortex mixer (Maxi Mix II, Thermolyne). The Bound-EPS was extracted following the heat extraction method (Morgan et al., 1990), in which the re-suspended sludge was heated to $50^{\circ} \mathrm{C}$ for 25 min in a water bath. The solid 
matter was then separated by centrifugation at 4,000 g for $15 \mathrm{~min}$, and the centrate was collected as the Bound-EPS extract. The sludge cake layer deposited on the membrane module was also collected from the MBR. When the membrane was severely fouled, the cake sludge (CS) was scraped off using a spatula. The recovered CS was re-suspended and dispersed by stirring it into a $0.05 \% \mathrm{NaCl}$ solution to an MLSS concentration of around 5 $\mathrm{g} / \mathrm{L}$. The CS supernatant was collected after sedimentation for $12 \mathrm{~h}$ at $4^{\circ} \mathrm{C}$, and the organic matter in the supernatant was further characterized.

\subsection{Evaluation of the fouling propensity of the MBR sludge}

The membrane-fouling propensity of the MBR sludge was evaluated using a singlefiber microfiltration (MF) apparatus (Sun et al., 2010). The fouling test apparatus was made of a plexiglass tube $1.5 \mathrm{~cm}$ in diameter and $50 \mathrm{~cm}$ in height. A polyethylene hollow-fiber MF membrane $\left(0.4 \mu \mathrm{m}\right.$, surface area $=16 \mathrm{~cm}^{2}$, Mitsubishi Rayon $)$ was installed vertically along the centerline of the tube. The sludge suspension was pumped (SELTZ-L40 II, Hydor) in circulation from and back to a feed tank to generate a cross flow through the test tube, and the permeate of the membrane filtration was withdrawn by a suction pump (MasterFLEX, ColeParmer). The cross-flow rate and filtration flux were set to $2 \mathrm{~L} / \mathrm{min}(0.19 \mathrm{~m} / \mathrm{s})$ and $37.5 \mathrm{~L} / \mathrm{m}^{2}$ h, respectively. An electronic balance (Arrw 60, OHAUS) was employed to record the permeate production, and the permeate was returned to the feed tank unless sampled for analysis. A pressure sensor (PTX Ex-0129, Druck) was installed before the suction pump to record the TMP variation. The membrane-fouling rate was measured as the increase in TMP for the amount of permeate produced (filtrate depth, L), or $\Delta \mathrm{TMP} / \Delta \mathrm{L}$.

The MF fouling test was conducted around every ten days on the MBR sludge with a main focus on the effect of the liquid-phase property on the fouling propensity of the sludge mixture. The MBR sludge was collected from the reactor, and its solid phase and liquid phase 
were separated by sedimentation at $4^{\circ} \mathrm{C}$ for up to $4 \mathrm{~h}$. Activated sludge suspension was collected from the ASP reactor, and its supernatant obtained by sedimentation. The MBR sludge sediment and the MBR supernatant were then diluted in tap water to form a sludge mixture with pre-determined MLSS and organic concentrations for the fouling test. For comparison of the liquid-phase effect, the same MBR sludge sediment and ASP supernatant were mixed and diluted in tap water to make a different sludge mixture. To ensure that the fouling test results were comparable throughout the two experimental stages, each of the sludge mixture samples for the MF fouling test had an identical MLSS concentration (3 g/L) and a fixed TOC (3.5 mg/L) in the liquid phase.

\subsection{Analytical methods}

The suspended solids concentration and the 30-min sludge volume index $\left(\mathrm{SVI}_{30}\right)$ were measured in accordance with the Standard Methods (APH-AWWA-WEF, 1998). The TOC concentration of the liquid samples was determined by a TOC analyzer (IL550 TOC-TN Analyzer, Lachat) using the high-temperature combustion method. The turbidity and viscosity of the sludge supernatant were measured by a turbidity meter (2100 P, HACH) and a vibration viscometer (SV-10, A\&D, Japan), respectively. For the liquid samples, including the Bound-EPS extract, the proteins and humic substances were measured by a UV/VIS spectrophotometer (Lambda 25, Perkin Elmer) following the modified Lowry method using bovine serum albumin (Sigma) and humic acid (Fluka) as the standards (Frǿlund et al., 1995), respectively. The polysaccharide content was determined by the phenol-sulfuric acid method using glucose as the standard (Gerhardt et al., 1994).

The organic materials in the sludge supernatant samples were filtered through a 0.4$\mu$ m polycarbonate membrane (25 mm, Osmonics). The organics in the filtrate were SMP, and the organics collected on the filter were defined as BPC. In addition to the concentration 
measurements, the size and chemical composition of the organic substances in the sludge supernatant were also characterized. The size distribution of the organic solutes was determined by a laser diffraction particle analyzer (LS 13 320, Beckman Coulter). Before the measurement, the organics in the sample were stained at a concentration of $20 \mathrm{mg} / \mathrm{L}$ with NanoOrange (Molecular Probes, Eugene), which is a fluorescent probe that targets the proteins in organic substances that may otherwise be transparent and non-detectable. Organic solutes larger than $0.4 \mu \mathrm{m}$, or BPC, were detected with the particle size analyzer (Sun et al., 2008). The BPC collected on the filters were also examined with a confocal laser scanning microscope (CLSM) (LSM Pascal, Zeiss, Thornwood, NY), following the procedures described previously (Chu and Li, 2005; Yang et al., 2008). For the CLSM observations, the BPC in the sludge supernatant were filtered on a $0.4-\mu \mathrm{m}$ black polycarbonate membrane (25 mm, Osmonics). The filtered samples were then stained using a combination of two probes: SYTO9 to target bacterial cells and ConA-TRITC to target polysaccharides with D-glucose or D-mannose (Sun et al., 2008).

\section{Results and Discussion}

\subsection{Sludge liquid phase variation}

During the experimental studies with either the MBR sludge or ASP sludge as the seed biomass, both the MBR and ASP systems performed well in terms of wastewater treatment. The organic removal efficiency as measured by the TOC reduction averaged more than $95 \%$ for the ASP and more than 97\% for the MBR (Table 1). As described previously, the two comparative reactors were operated side by side under similar conditions in terms of the HRT, SRT, and MLSS concentrations and the F/M ratio. The main difference between the MBR and ASP lay in the method of sludge-effluent separation. As a result, different trends of 
change in the liquid-phase property were observed for the sludge in the two reactors. In Experiment I in which the MBR sludge was used as the seed biomass, the organic concentration remained at a high level of $10 \mathrm{mg}$ TOC/L or more in the supernatant of the MBR sludge (Fig. 1). In contrast, the TOC in the supernatant of the ASP sludge decreased gradually from about $15 \mathrm{mg} / \mathrm{L}$ to a level of below $4 \mathrm{mg} / \mathrm{L}$. With membrane filtration, the MBR effluent had a lower organic concentration than the sludge supernatant, which is a typical feature of MBR systems (Chu and Li, 2005; Rosenberger et al., 2005). On average, the effluent TOC was less than 50\% of the organic in the MBR supernatant. For the ASP using sedimentation to achieve sludge-effluent separation, the organic concentration in the effluent was largely identical to that in the sludge supernatant, as expected.

In Experiment II, activated sludge was used as the seed biomass. The concentration of organics in both the MBR and ASP increased from less than 5 to around $15 \mathrm{mg} / \mathrm{L}$ on the first day of startup. This initial increase was probably caused by the transport of the sludge and sudden changes in the reactor environment and the feed substrate to the sludge. It took a few days for the biomass to acclimatize to the laboratory bioreactors, and thus the TOC concentration dropped to a low level in the first $10 \mathrm{~d}$ of operation. Thereafter, despite a certain degree of fluctuation in TOC, the organic concentration showed a trend of increase to more than $10 \mathrm{mg} / \mathrm{L}$ in the MBR supernatant. In comparison, the TOC decreased to a lower level of less than $5 \mathrm{mg} / \mathrm{L}$ in the ASP sludge supernatant (Fig. 1). These different change trends in the liquid-phase organic content of the two sludge types agree well with previous comparisons of MBR and ASP processes during startup (Shin and Kang, 2003; Masse et al., 2006). Compared with sedimentation, membrane filtration in an MBR not only separates sludge flocs, but also removes organic matter from the effluent, resulting in a higher level of organic content in the MBR suspension. In other words, whereas ASP allows the washout of organic from the system, the MBR retains organic matter within the reactor. 
The change in the liquid-phase property of the sludge in the MBR was also indicated by other parameters, such as the turbidity and viscosity. During Experiment I, which involved seeding with MBR sludge, the turbidity of the sludge supernatant from the MBR remained nearly unchanged at around 20 NTU, whereas the turbidity of the ASP supernatant gradually decreased to 3 NTU or lower (Fig. 2). Apparently, the ASP transformed the MBR seed sludge into a typical activated sludge mixture with a reduced amount of turbidity-causing impurities in the supernatant. In Experiment II, which involved seeding with activated sludge, the turbidity of the sludge supernatant from the ASP showed little change during the experiment. However, the turbidity of the MBR supernatant increased gradually from less than 2 NTU to more than 16 NTU (Fig. 2). Compared with the ASP sludge, the organic matter in the MBR sludge not only increased in concentration after membrane filtration, but also changed to a form that caused greater turbidity. Similar changes were observed for the viscosity of the sludge supernatants from the MBR and ASP. When the MBR sludge was used as the seed sludge, the viscosity of the sludge supernatant stayed at a high level of above $1.1 \mathrm{mPa}-\mathrm{s}$ in the MBR, but decreased to around $1.0 \mathrm{mPa}-\mathrm{s}$ in the ASP. When the ASP sludge was used as the seed sludge, the viscosity of the MBR supernatant increased from around 1.01 to more than $1.1 \mathrm{mPa}-\mathrm{s}$, whereas the viscosity of the ASP supernatant remained at a low level of around 1.0 mPa-s. The higher viscosity of the MBR sludge compared with the ASP sludge mixture implies the formation of more viscous solutes in the sludge suspension in the former.

With the glucose-based substrate used in this study, the organic residue in the sludge mainly consists of SMP. Moreover, a pool of free and large organic solutes, or BPC, is also expected in MBR sludge suspension (Wang et al., 2007; Sun et al., 2008; Lin et al., 2009). Distinct from sludge flocs, BPC are composed mainly of organic polymers rather than microbial cells (Sun et al., 2008). In the ASP sludge, the SMP actually contributed little to the turbidity and viscosity of the sludge supernatant. In contrast, BPC formation and 
accumulation in the MBR increased both the turbidity and viscosity of the sludge supernatant considerably (Fig. 2). Despite the changes in the liquid-phase property, the changes in the solid-phase property of the sludge were rather minimal according to the analysis of the biomass Bound-EPS. The EPS content was rather stable and largely comparable between the ASP and MBR sludge during the two experiments with the different types of seed sludge (Fig. S1, Supplementary Data). Hence, the observed change in sludge fouling potential did not appear to be closely related to the EPS content of the sludge, which is different from some of previous reports (Nagaoka et al., 1996; Wang et al., 2010; Wu and Lee, 2011).

\subsection{Effect of the liquid-phase property on the fouling propensity of MBR sludge}

The effect of the liquid-phase property on the fouling propensity of the MBR sludge was evaluated using a single-fiber MF apparatus. The settled MBR sludge was diluted into either the MBR supernatant solution or the ASP supernatant solution to make a new sludge mixture for the MF fouling test. At a predetermined filtration flux $\left(37.5 \mathrm{~L} / \mathrm{m}^{2}-\mathrm{h}\right)$ and crossflow velocity $(0.19 \mathrm{~m} / \mathrm{s})$, the rate of TMP increase during sludge filtration was used to compare the fouling propensity of the different sludge mixture samples. In both Experiments I and II, the profiles of the membrane-fouling rate were different for the MBR sludge mixed with the MBR supernatant and that mixed with the ASP supernatant. In Experiment I, the MBR sludge suspension had a high fouling potential throughout the experiment, and the rate of TMP increase determined by the single-fiber MF test remained in the range of 80 to 100 $\mathrm{kPa} / \mathrm{m}$. However, when the MBR supernatant was replaced by the counterpart ASP supernatant, the fouling propensity of the sludge mixture was considerably reduced. The corresponding TMP increase rate decreased from more than $100 \mathrm{kPa} / \mathrm{m}$ to below $50 \mathrm{kPa} / \mathrm{m}$ after $25 \mathrm{~d}$ of operation. In Experiment II, in which ASP sludge was used as the seed biomass, the sludge mixture had a low initial membrane fouling potential. The fouling propensity of 
the MBR sludge mixture (MBR sludge and MBR supernatant) then increased continuously with time as its TMP increase rate grew from about 75 to over $100 \mathrm{kPa} / \mathrm{m}$ after $45 \mathrm{~d}$. However, when the settled MBR sludge was mixed with the ASP supernatant, the fouling potential of the sludge mixture dropped. The MBR sludge and ASP supernatant mixture exhibited a low fouling potential, with a TMP increase rate of less than $60 \mathrm{kPa} / \mathrm{m}$ in the late phase of Experiment II.

The fouling propensity of the MBR sludge mixture in Experiment II eventually became rather similar to that recorded for the MBR sludge in Experiment I, despite the different seed sludge used. The mixture of the MBR sludge and ASP supernatant also displayed a comparable fouling propensity between the two experiments after $45 \mathrm{~d}$ of startup. The fouling potential of the sludge in the MBR that was seeded with activated sludge increased with time, suggesting a gradual change from a typical ASP sludge to an MBR sludge. Most of the change was brought about in the liquid phase, rather than the solid phase, of the sludge suspension. The liquid-phase property had a profound effect on the fouling propensity of the sludge. For the same MBR sludge, when the supernatant was replaced by the ASP supernatant, the fouling rate of the sludge mixture was greatly reduced.

The main difference between the ASP and MBR sludge supernatants was further revealed by microscopic examination (Fig. 3). According to the CLSM images, there were few cells or polysaccharide organics in the ASP supernatant (Fig. 3a). Most of the organic residues in the ASP supernatant were expected to be SMP, which are too small to be intercepted by the MF filter. In contrast, much large organic matter was found in the MBR supernatant, and few cells were present (Fig. 3b). This indicates the formation and accumulation of BPC in the MBR suspension, some of which were larger than $10 \mu \mathrm{m}$. Large BPC have been identified as serious foulants in MBR sludge mixture (Wang et al., 2007; Sun et al., 2008; Lin et al., 2009). BPC interact with biomass flocs to play an important role in the 
deposition of the sludge layer on the membrane surface, resulting in progressive membrane fouling. More abundant BPC were found in the sludge cake layer, as shown by the large BPC in the supernatant of the cake sludge collected from the MBR (Fig. 3c).

The size evolution of the organic solutes in the sludge from the different reactors was further confirmed by size distribution measurements (Fig. 4). By using NanoOrange to stain the proteins in the organic polymers, BPC larger than $0.4 \mu \mathrm{m}$ in the sludge supernatant samples were made visible to the Coulter laser diffraction particle analyzer (Wang et al., 2007; Sun et al., 2008). The seed MBR sludge in Experiment I contained a large portion of BPC that reached several tens of micrometers in size. However, due to the washout mechanism of the ASP, the sludge in the reactor gradually lost the characteristics of the seed MBR sludge, and the BPC in ASP supernatant became much smaller in size. The average size of the organic solutes decreased from $8.5 \mu \mathrm{m}$ to about $1.1 \mu \mathrm{m}$ after $32 \mathrm{~d}$ of ASP operation. At the same time, as shown in Fig. 1, the organic concentration also decreased considerably and BPC became less abundant in the ASP suspension. In Experiment II, in which ASP sludge was used as the seed, BPC formation and accumulation in the MBR sludge was also evident. The average size of the organic solutes in the MBR supernatant increased from about $0.78 \mu \mathrm{m}$ to $7.9 \mu \mathrm{m}$ after $38 \mathrm{~d}$ (Fig. 4). Hence, the seed ASP sludge was transformed by the MBR to a typical MBR sludge with a higher content of large BPC.

\subsection{BPC formation and its impact on membrane fouling in the $M B R$}

The experimental results indicate the formation and growth of BPC in the MBR system but not in the ASP. The primary difference between an ASP and MBR lies in the solid-liquid separation step. SMP are ubiquitously present in activated sludge as poorly biodegradable organic residues of biological origin (Laspidou and Rittmann, 2002). In an ASP, SMP readily flow out of the system with the effluent from the sedimentation tank. In an 
MBR, a major portion of the SMP may also escape from the system with the effluent, as the membrane pore sizes are usually only within a range to retain (sieve) sludge flocs other than organic molecules. However, adsorption of SMP on the membrane and, more importantly, by the sludge layer on the membrane surface, is inevitable (Yamato et al., 2006; Drews et al., 2008; Xiao et al., 2009; Wu and Lee, 2011). The continuous adsorption and accumulation provides an opportunity for the SMP in the sludge layer to attach to each other and grow into larger organic clusters, or BPC. However, the shear forces caused by aeration continuously detach organic matter (SMP and BPC) and the sludge from the membrane surface, bringing organic solutes into the MBR sludge suspension (Wang et al., 2007; Sun et al., 2008). Apparently, when BPC are formed, they have little chance of escaping from the enclosed MBR system, but instead accumulate and grow. However, the repeated adsorption and shear detachment of BPC break up large BPC into smaller components, thereby keeping the BPC to within a certain size range in the sludge mixture.

The BPC concentration in the MBR suspension also appeared to have an upper limit (Fig. 1). The extended retention of BPC by membrane filtration gives rise to greater BPC degradation in the MBR. As described earlier, the other sink for BPC in an MBR is their involvement in sludge cake formation on the membrane surface. A thorough comparison is made that shows the organic concentration normalized over the sludge concentration (i.e. TOC/SS) in different phases of the experiment (Fig. S2, Supplementary Data). In the later stage of the reactor startup, the MBR supernatant had a higher organic content than its counterpart ASP supernatant. Nearly half of the organic substances in the MBR sludge were BPC. The MBR cake sludge had a much higher BPC content (18.6 mg TOC/g SS) than the bulk sludge (2.1 mg TOC/ g SS). As shown by the CLSM images, the BPC trapped within the sludge layer were much larger (average size $=32.4 \mu \mathrm{m}$ ) than the BPC in the sludge suspension (Fig. 3). The accumulation of large BPC in the sludge cake layer on the 
membrane surface is believed to be mainly responsible for the great filtration resistance of the cake layer in an MBR (Wang et al., 2007; Sun et al., 2008). Although the EPS content of sludge was thought to be crucial to the fouling propensity of the sludge (Nagaoka et al., 1996; Wang et al., 2010; Wu and Lee, 2011), the present results suggest that BPC formation and growth are more important to the membrane fouling development in MBR.

The BPC degradation and transformation dynamics in different phases of the MBR system in Experiment II were further analyzed. There were four operational cycles between membrane cleanings for the startup period of around $70 \mathrm{~d}$ (Fig. 5). For each cycle, the amount of organics retained by membrane filtration in the MBR was calculated based on the volume of permeate (effluent) production and the TOC difference between the MBR supernatant and its effluent. The total amount of TOC removed by membrane filtration in each cycle ranged from 1,712 to 2,333 mg with an average of around 2,000 mg. The total BPC trapped within the sludge cake layer was up to $200 \mathrm{mg}$ TOC, which accounted for about $10 \%$ of the organic matter retained (Fig. 5). The amount of organic solutes in the MBR suspension was around $60 \mathrm{mg}$. Hence, more than $85 \%$ of the SMP and other organic matter intercepted by membrane filtration were apparently removed by biological degradation in the MBR. Most of the rest formed BPC and accumulated within the sludge cake layer. In general, the BPC accumulation in the cake sludge correlated well with the TMP increase and fouling development in the MBR system.

The experimental results demonstrate that membrane filtration in the MBR intercepted SMP and kept them within the reactor. More importantly, the vast membrane surface facilitated the transformation of organic substances from SMP to more detrimental foulants, that is, BPC. A submerged MBR is basically an enclosed system that does not allow the washout or discharge of organic foulants from the sludge suspension. As a result, foulants accumulate in the system, either in the suspension or within the sludge cake. Thus, it is 
membrane filtration that largely gives rise to the MBR fouling problem. For BPC formation and growth, the precursors (e.g., SMP) must be sticky with a high affinity feature such that

they have a strong tendency to cluster on the membrane surface. Among the three major components measured for organic solutes, humic substances had a lower membrane retention ratio compared with proteins and polysaccharides, which is in agreement with previous findings (Al-Halbouni et al., 2009). The humic content increased from $38 \%$ to $28 \%$ in the liquid phase of the MBR sludge during Experiment II and decreased from 28\% to 37\% in the liquid phase of the ASP sludge during Experiment I (Fig. S2, Supplementary Data). Proteins and polysaccharides made greater contributions at 33\% and 39\%, respectively, than humic substances to BPC formation in the MBR system.

\section{Conclusions}

Compared with ASP, MBR sludge has a more BPC in the liquid-phase, which are formed and retained by membrane filtration. The liquid-phase property of sludge suspension has a profound impact on the sludge fouling propensity. The BPC dynamics in MBR involve not only a concentration increase but also size growth. A submerged MBR is basically an enclosed system that concentrates organic foulants in the sludge suspension. The membrane surface and overlying sludge layer provide a unique environment for the transformation of organic solutes from SMP to BPC, leading to further sludge cake deposition and serious membrane fouling.

\section{Acknowledgments}

This research was supported by URC funding from the University of Hong Kong, Special Equipment Grant SEG_HKU10 from the University Grants Council (UGC), and 
Grant HKU7144/E07 from the Research Grants Council (RGC) of the Hong Kong SAR

Government. The technical assistance of Mr. Keith C. H. Wong is greatly appreciated.

\section{References}

AEESP, 2001. Environmental Engineering Process Laboratory Manual. Association of Environmental Engineering and Science Professors, Champaign, IL, USA.

Al-Halbouni, Dott, W., Hollender, J., 2009. Occurrence and composition of extracellular lipids and polysaccharides in a full-scale membrane bioreactor. Water Res. 43, 97-106. APHA-AWWA-WEF, 1998. Standard Methods for the Examination of Water and Wastewater, 20th ed. American Public Health Association/American Water Works Association/Water Environment Federation, Washington, DC, USA.

Chu, H.P., Li, X.Y., 2005. Membrane fouling in a membrane bioreactor (SMBR): Sludge cake formation and fouling characteristics. Biotechnol. Bioeng. 90, 323-331.

Defrance, L., Jaffrin, M.Y., Gupta, B., Paullier, P., Geaugey, V., 2000. Contribution of various constituents of activated sludge to membrane bioreactor fouling. Bioresource Technol. 73(2), 105-112.

Drews, A., Vocks, M., Bracklow, U., Iversen, V., Kraume, M., 2008. Does fouling in MBRs depend on SMP? Desalination, 231(1-3), 141-149.

Drews, A., 2010. Membrane fouling in membrane bioreactors - Charactersation, contradiction, cuse and cures. J. Membr. Sci. 373, 1-28.

Frǿlund, B., Griebe, T., Nielsen, P.H., 1995. Enzymatic-activity in the activated-sludge floc matrix. Appl. Microbiol. Biotechnol. 43, 755-761.

Gerhardt, P., Murray, R.G.E., Wood, W.A., Krieg, N.R., 1994. Methods for general and molecular bacteriology, American Society for Microbiology, Washington, DC, USA. 
Ji, L., Zhou, J., 2006. Influence of aeration on microbial polymers and membrane fouling in submerged membrane bioreactors, J. Membr. Sci. 276, 168-177.

Judd, S., 2006. The SMBR Book: Principles and Applications of Membrane Bioreactors in Water and Wastewater Treatment. Elsevier, Amsterdam, The Netherlands.

Judd, S., Jefferson, B., 2003. Membranes for Industrial Wastewater Recovery and Re-use. Elsevier, Oxford, UK.

Laspidou, C.S., Rittmann B.E., 2002. A unified theory for extracellular polymeric substances, soluble microbial products, and active and inert biomass. Water Res. 36, 2711-2720.

Le-Clech, P., Chen, V., Fane, T.A.G., 2006. Fouling in membrane bioreactors used in wastewater treatment. J. Membr. Sci. 284, 17-53.

Li, X.Y., Wang, X.M., 2006. Modelling of membrane fouling in a submerged membrane bioreactor. J. Membr. Sci. 278, 151-161.

Lin, H.J., Xie, K., Mahendran, B., Bagley, D.M., Leung, K.T., Liss, S.N., Liao, B.Q., 2009. Sludge properties and their effects on membrane fouling in submerged anaerobic membrane bioreactors (SAnMBRs). Water Res. 43, 3827-3837.

Magara, Y., Itoh, M., 1991. The effect of operational factors on solid/liquid separation by ultra-membrane filtration in a biological denitrification system for collective human excrete treatment plant. Water Sci. Technol. 23(7/8/9), 1583-1590.

Masse, A., Sperandio, M., Cabassud, C., 2006. Comparison of sludge characteristics and performance of a submerged membrane bioreactor and an activated sludge process at high solids retention time. Water Res. 40, 2405-2415.

Meng, F.G., Chae, S.-R., Drew, A., Kraume, M., Shin, H.-S., Yang, F., 2009. Recent advances in membrane bioreactors (MBRs): Membrane fouling and membrane material. Water Res. 43, 1489 -1512. 
Morgan, J.W., Forster, C.F., Evison, L., 1990. A comparative-study of the nature of biopolymers extracted from anaerobic and activated sludges. Water Res. 24, 743-750.

Nagaoka, H., Ueda, S., Miya A., 1996. Influence of bacterial extracellular polymers on the membrane separation activated sludge process. Water Sci. Technol. 34(9), 165-172.

Rosenberger, S., Evenblij, H., Poele, S.T., Wintgens, T., Laabs, C., 2005. The importance of liquid phase analyses to understand fouling in membrane assisted activated sludge processes - Six case studies of different european research groups. J. Membr. Sci. 263, $113-126$.

Shin, H.-S., Kang, S.-T., 2003. Characteristics and fates of soluble microbial products in ceramic membrane bioreactor at various sludge retention times. Water Res. 37, 121-127.

Sun, F.Y., Wang, X.M. and Li, X.Y. 2008 Visualisation and characterisation of biopolymer clusters in a submerged membrane bioreactor. J. Membr. Sci. 325: 691-697.

Sun, F.Y., Wang, X.M. and Li, X.Y. 2011 Effect of biopolymer clusters on the fouling property of sludge from a membrane bioreactor (MBR) and its control by ozonation. Process Biochem. 46, 162-167

Wang, X., Zhang, B., Shen, Z., Qiu, Z., Chen, Z., Jin, M., Li J., Wang, J., 2010. The EPS characteristics of sludge in an aerobic granule membrane bioreactor. Bioresour. Technol. 101, 8046-8050.

Wang, X.M., Li, X.Y., Huang, X., 2007. Membrane fouling in a submerged membrane bioreactor (SMBR): Characterisation of the sludge cake and its high filtration. Sep. Purif. Technol. 52, 439-445.

Wang, X.M., Li, X.Y., 2008. Accumulation of biopolymer clusters in a submerged membrane bioreactor and its effect on membrane fouling. Water Res. 42, 855-862. 
Wu, S.C., Lee, C.M. 2011. Correlation between fouling propensity of soluble extracellular polymeric substances and sludge metabolic activity altered by different starvation conditions. Bioresour. Technol. doi: 10.1016/j. biortech. 2010.11.093.

Xiao, K., Wang, X.M., Huang, X., Waite, T.D., Wen, X.H., 2009. Analysis of polysaccharide, protein and humic acid retention by microfiltration membranes using Thomas' dynamic adsorption model. J. Membr. Sci. 342, 22-34.

Yamato, N., Kimura, K., Miyoshi, T., Watanabe, Y., 2006. Difference in membrane fouling in membrane bioreactors (MBRs) caused by membrane polymer materials. J. Membr. Sci. 280, 911-919.

Yang, S.F., Li, X.Y., Yu, H.Q., 2008. Formation and characterisation of fungal and bacterial granules under different feeding alkalinity and $\mathrm{pH}$ conditions. Process Biochem. 43, 814.

Yang, X., Song, H., Lu, J., Fu, D., Cheng, B., 2010. Influence of diatomite addition on membrane fouling and performance in a submerged membrane bioreactor. Bioresour. Technol. 101, 9178-9184. 
Table 1. Overall wastewater treatment performance of the MBR and ASP systems.

\begin{tabular}{lllll}
\hline Experiment & \multicolumn{2}{c}{ I } & \multicolumn{2}{c}{ II } \\
\hline Reactor system & \multicolumn{1}{c}{ MBR } & \multicolumn{1}{c}{ ASP } & \multicolumn{1}{c}{ MBR } & \multicolumn{1}{c}{ ASP } \\
\hline Seed sludge & MBR sludge & MBR sludge & ASP sludge & ASP sludge \\
Duration (d) & 68 & 68 & 73 & 73 \\
F/M (g TOC/g SS-d) & $0.121 \pm 0.015$ & $0.124 \pm 0.017$ & $0.125 \pm 0.021$ & $0.128 \pm 0.02$ \\
HRT(h) & 6 & 6 & 6 & 6 \\
SRT(d) & $9 \pm 1$ & $9 \pm 1$ & $9 \pm 1$ & $9 \pm 1$ \\
TOC removal (\%) & $97.4 \pm 1.8$ & $95.2 \pm 2.5$ & $98.7 \pm 1.3$ & $96.9 \pm 2.1$ \\
MLSS (g/L) & $4.6 \pm 0.5$ & $4.8 \pm 0.8$ & $5.1 \pm 0.6$ & $4.7 \pm 0.6$ \\
SVI 30 (mL/g) & $103.2 \pm 12.5$ & $131.5 \pm 15.1$ & $105.4 \pm 9.6$ & $128.5 \pm 10.3$ \\
Effluent SS (mg/L) & $<1$ & $80 \pm 27$ & $<1$ & $60 \pm 18$ \\
\hline \hline
\end{tabular}




\section{Figure Captions}

Fig. 1. Change in the organic concentrations in the sludge supernatant and effluent in the MBR and ASP during Experiment I seeded with MBR sludge and Experiment II seeded with ASP sludge.

Fig. 2. Change in turbidity and viscosity of the supernatants of the sludge from the MBR and ASP during Experiments I and II.

Fig. 3. CLSM images of the BPC in the liquid phase of the sludge samples: (a) ASP sludge on d 52 of Experiment I, (b) MBR sludge on d 38 of Experiment II, and (c) cake sludge obtained from the membrane surface of the MBR on d 55 of Experiment II. (red: polysaccharides in BPC; green: bacteria)

Fig. 4. Evolution of the BPC size distribution in the sludge supernatant from the ASP during Experiment I seeded with the MBR sludge and from the MBR during Experiment II seeded with the ASP sludge.

Fig. 5. (a) TMP recorded for the MBR during Experiment II; (b) amount of organics retained by membrane filtration and amount of BPC trapped in the cake sludge of the MBR. 




Fig. 1. Change in the organic concentrations in the sludge supernatant and effluent in the MBR and ASP during Experiment I seeded with MBR sludge and Experiment II seeded with ASP sludge. 


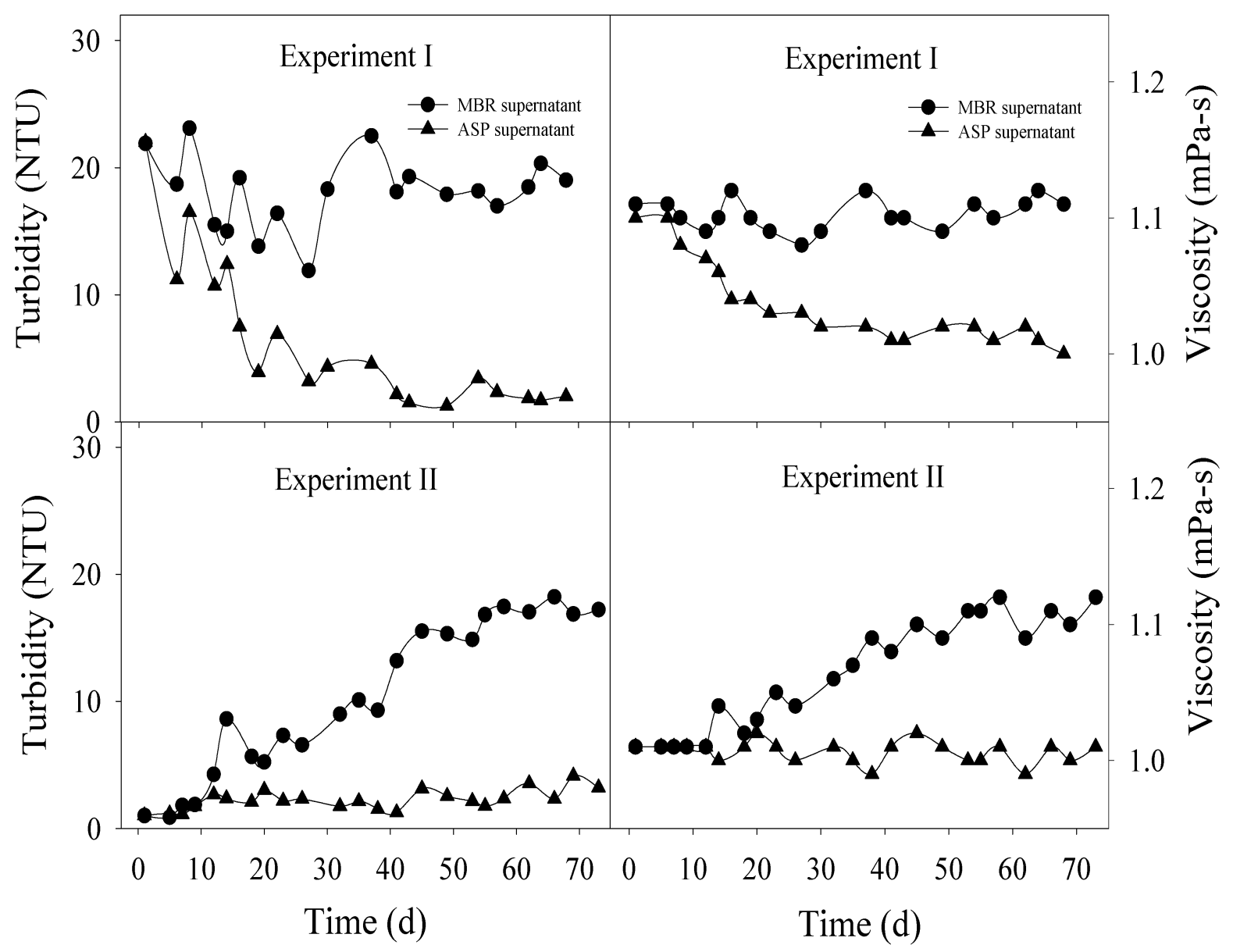

Fig. 2. Change in turbidity and viscosity of the supernatants of the sludge from the MBR and ASP during Experiments I and II. 

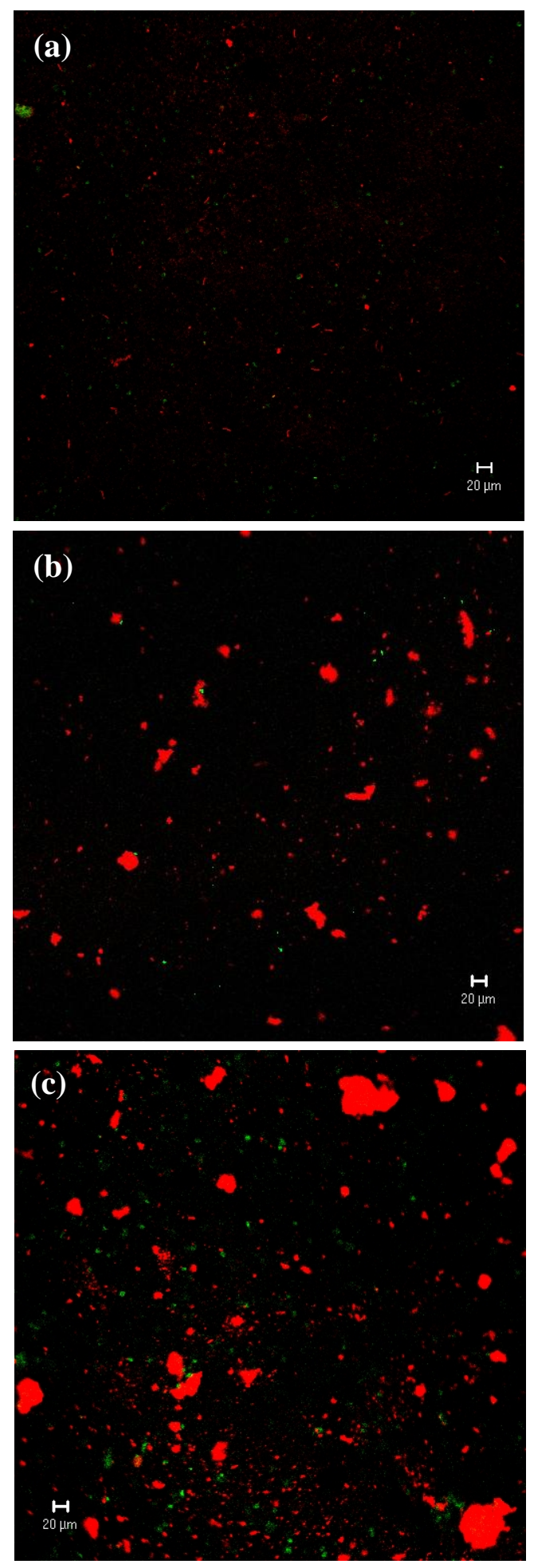

Fig. 3. CLSM images of the BPC in the liquid phase of the sludge samples: (a) ASP sludge on d 52 of Experiment I, (b) MBR sludge on d 38 of Experiment II, and (c) cake sludge obtained from the membrane surface of the MBR on d 55 of Experiment II. (red: polysaccharides in BPC; green: bacteria) 


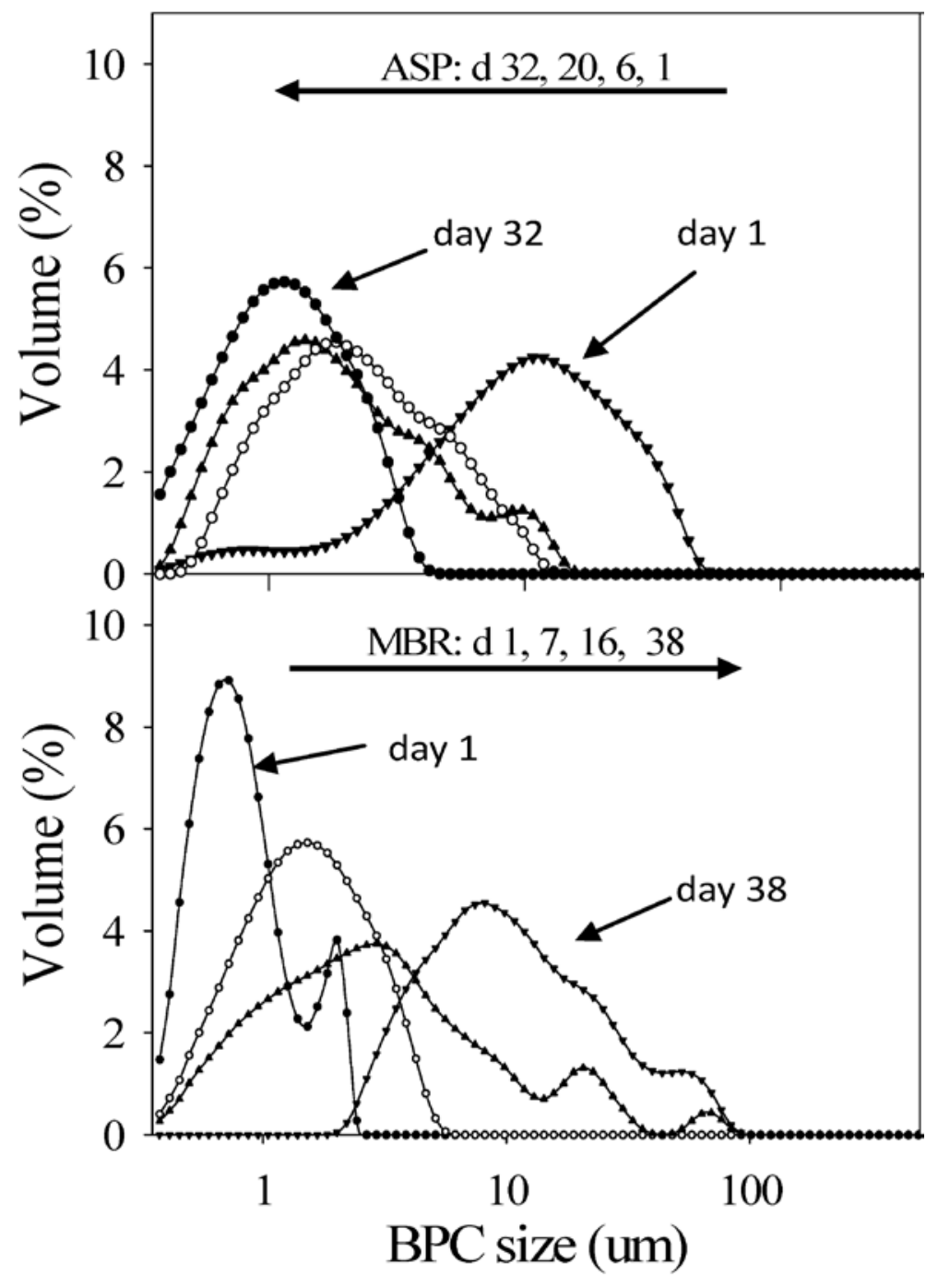

Fig. 4. Evolution of the BPC size distribution in the sludge supernatant from the ASP during Experiment I seeded with the MBR sludge and from the MBR during Experiment II seeded with the ASP sludge. 




Fig. 5. (a) TMP recorded for the MBR during Experiment II; (b) amount of organics retained by membrane filtration and amount of BPC trapped in the cake sludge of the MBR. 